\title{
Phosphorus ion implantation in silicon nanocrystals embedded in $\mathrm{SiO}_{\mathbf{2}}$
}

\author{
Kouichi Murakami, ${ }^{1, a)}$ Ryota Shirakawa, ${ }^{1}$ Masatoshi Tsujimura, ${ }^{1}$ Noriyuki Uchida, ${ }^{2}$ \\ Naoki Fukata, ${ }^{3}$ and Shun-ichi Hishita ${ }^{4}$ \\ ${ }_{1}^{1}$ Institute of Applied Physics, University of Tsukuba, Tennodai 1-1-1, Tsukuba 305-8577, Japan \\ ${ }^{2}$ Nanodevice Innovation Research Center, Advanced Industrial Science and Technology (AIST), \\ Higashi 1-1-1, Tsukuba, Ibaraki 305-8562, Japan \\ ${ }^{3}$ International Center for Materials Nanoarchitectonics (MANA), National Institute for Materials Science \\ (NIMS) and JST PRESTO, 1-1 Namiki, Tsukuba 305-0044, Japan \\ ${ }^{4}$ Sensor Materials Center, National Institute for Materials Science (NIMS), 1-1 Namiki, Tsukuba, Ibaraki \\ 305-0044, Japan
}

(Received 29 May 2008; accepted 23 January 2009; published online 10 March 2009)

\begin{abstract}
We have investigated phosphorus ion $\left(\mathrm{P}^{+}\right)$implantation in $\mathrm{Si}$ nanocrystals (SiNCs) embedded in $\mathrm{SiO}_{2}$, in order to clarify the P donor doping effects for photoluminescence (PL) of SiNCs in wide $\mathrm{P}$ concentrations ranging in three orders. Some types of defects such as $\mathrm{P}_{b}$ centers were found to remain significantly at the interfaces between $\mathrm{SiNCs}$ and the surrounding $\mathrm{SiO}_{2}$ even by high-temperature $\left(1000{ }^{\circ} \mathrm{C}\right)$ annealing of all the samples. Hydrogen atom treatment (HAT) method can efficiently passivate remaining interface defects, leading to significant increase in the intensity of PL arising from the recombination of electron-hole pairs confined in SiNCs, in addition to significant decrease in interface defects with dangling bonds detected by electron spin resonance. From both the results of the P dose dependence before and after HAT, it is found that the amount of remaining defects is higher for samples with SiNCs damaged by implantation with relatively lower $\mathrm{P}^{+}$doses and then annealed, and that through HAT the observed PL intensity increases surely as the $\mathrm{P}$ concentration increases up to a critical concentration. Then it begins to decrease due to Auger nonradiative recombination above the critical concentration which depends on the size of SiNCs. These results suggest an effect of relatively low concentration of $\mathrm{P}$ atoms for the enhancement of PL intensity of SiNCs and we present an unconventional idea for explaining it.

(C) 2009 American Institute of Physics. [DOI: 10.1063/1.3088871]
\end{abstract}

\section{INTRODUCTION}

Light emitting silicon $(\mathrm{Si})$ nanostructures such as porous $\mathrm{Si}$ and $\mathrm{Si}$ nanocrystals (SiNCs) or Si nanodots embedded in $\mathrm{SiO}_{2}$ matrices have been attractive for the optoelectronics application ${ }^{1}$ and nonvolatile memory devices, ${ }^{2}$ while the present Si-LSI technology has advanced into the nanoscale sizes less than $100 \mathrm{~nm}$ for more than 10 years. It has been already understood that quantum confinement is an origin for the blue shift photoemission from Si nanostructures, and that shallow level impurities such as phosphorus $(\mathrm{P})$ donor and boron (B) acceptor in bulk crystal Si have slightly deeper, but still shallow levels even in SiNCs less than approximately $10 \mathrm{~nm} .{ }^{3,4}$ For nanoelectronics using semiconductors, impurity doping will continue to be very important for control of carrier concentration leading to control of the electrical conductivity and for control of the efficiency of light emission such as photoluminescence (PL) and electroluminescence. ${ }^{1,2,5}$ While as an impurity doping technique, only ion implantation has been developed especially for Si-LSI processes since 1970s, it has been used also in formation of SiNCs in $\mathrm{SiO}_{2},{ }^{1-3}$ defect production, ${ }^{6}$ and impurity doping ${ }^{1}$ in SiNCs.

Fujii and co-workers ${ }^{3,7}$ reported P doping effects for PL intensity that light doping produces enhancement of PL for SiNCs with relatively small sizes, but not for those for larger

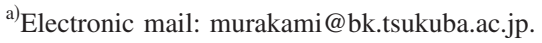

SiNCs, and heavy P doping leads to decrease in PL intensity. The latter is due to Auger nonradiative recombination. ${ }^{7}$ On the other hand, any clear explanations have been given for the former enhancement, although it has been reported that the decrease in the number of interface defects may be produced by light $\mathrm{P}$ doping. ${ }^{7}$

Since SiNCs embedded in $\mathrm{SiO}_{2}$ have very large interface-to-bulk ratio, the large difference between the $\mathrm{Si}-\mathrm{Si}$ atom bonding distance in the Si crystal cores and that of the surrounding $\mathrm{SiO}_{2}$ induces high density of interface defects. Thus, we have to investigate $\mathrm{P}$ doping effects for SiNCs embedded in $\mathrm{SiO}_{2}$ in which the interface defects are enough passivated. This is just the problem for Si nanostructutures because Si surface is easily oxidized. Annihilation of deep levels caused by the interface defects, or passivation of the defects, is needed also for development of the future Sinanostructure devices. One candidate of techniques for it is hydrogen passivation which has been intensively investigated for surface layers up to several microns of bulk $\mathrm{Si}^{8}{ }^{8}$ $\mathrm{Si} / \mathrm{SiO}_{2}$ interface, ${ }^{9}$ and $\mathrm{Si}$ surface.

In this study, we have used $\mathrm{P}^{+}$ion implantation in SiNCs formed in $\mathrm{SiO}_{2}$ matrices and a hydrogen passivation technique in order to clarify the P donor doping effects for PL of SiNCs with $\mathrm{P}$ concentrations ranging in three orders, much wider than those of the previous studies. ${ }^{3,7}$ For investigation of ion implantation effects in silicon $(\mathrm{Si})$ nanostructures, we have measured PL and electron spin resonance (ESR) of un- 


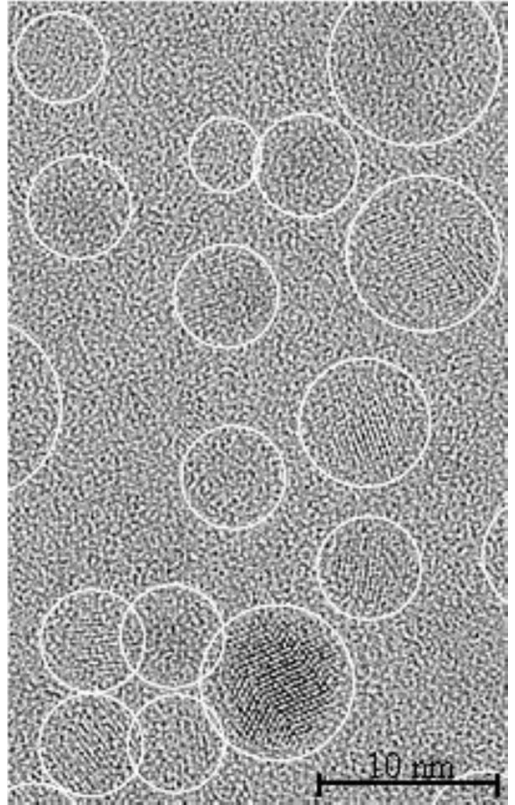

FIG. 1. Typical TEM image for a sample formed at $1200{ }^{\circ} \mathrm{C}$, showing SiNCs embedded in $\mathrm{SiO}_{2}$ by the dotted circulars.

doped SiNCs and $\mathrm{P}$ doped SiNCs embedded in $\mathrm{SiO}_{2}$ before and after hydrogen passivation. We call hereafter these systems $\mathrm{SiNCs} / \mathrm{SiO}_{2}$, for simplicity. For these experiments, first the samples of $\mathrm{SiNCs} / \mathrm{SiO}_{2}$ systems were grown by hightemperature annealing at 1000 or $1200{ }^{\circ} \mathrm{C}$ of $\mathrm{SiO}_{x}$ films deposited on a quartz glass. Second, for preparation of P-doped SiNCs, the $\mathrm{SiNCs} / \mathrm{SiO}_{2}$ were implanted with $\mathrm{P}^{+}$ion and then annealed at $1000{ }^{\circ} \mathrm{C}$, followed by an optimum hydrogen passivation to annihilate deep levels of defects at the $\mathrm{Si} / \mathrm{SiO}_{2}$ interfaces.

\section{EXPERIMENTAL PROCEDURE}

$\mathrm{SiO}_{x}$ films were deposited by thermal evaporation of $\mathrm{SiO}$. The thickness was approximately $500 \mathrm{~nm}$. $\mathrm{Si}^{+}$ion implantation in thermally oxidized $\mathrm{SiO}_{2}$ was also done to make another $\mathrm{SiNCs} / \mathrm{SiO}_{2}$ system. After the deposition or $\mathrm{Si}^{+}$ion implantation, thermal annealing was performed at 1000 and $1200{ }^{\circ} \mathrm{C}$, respectively, to attain SiNCs growth concomitantly with formation of $\mathrm{SiO}_{2}$ phase. The averaged diameter was estimated to be about 3-4 and 5-6 $\mathrm{nm}$ for the films formed at 1000 and $1200{ }^{\circ} \mathrm{C}$, respectively. These values were obtained from transmission electron microscopy (TEM) image measurements of some samples, as shown in Fig. 1 as a typical example (sample 2). Then phosphorus (P) ions were doubly implanted with the doses ranging from $10^{14}$ to $10^{17} / \mathrm{cm}^{2}$ at energies of 100 and $200 \mathrm{keV}$ to obtain nearly flat P impurity distribution in the depth direction, followed by thermal annealing of produced defects at $1000{ }^{\circ} \mathrm{C}$. PL at room temperature (RT) and ESR measurements at $4.2 \mathrm{~K}$ were performed before and after hydrogen atom treatment (HAT) ${ }^{10-12}$ i.e., hydrogen passivation of remaining defects such as $\mathrm{P}_{b}$ centers induced at the interface between SiNCs and the surrounding $\mathrm{SiO}_{2}$. HAT was done at $500{ }^{\circ} \mathrm{C}$ which was found to be an optimum temperature for efficient elimination of the interface defects. ${ }^{12}$ The samples prepared in this systematical study are summarized in Table I.
TABLE I. Samples prepared for P ion-implanted SiNCs.

\begin{tabular}{|c|c|c|c|c|}
\hline \multirow[b]{2}{*}{ Sample No. } & \multirow{2}{*}{$\begin{array}{l}\text { Formation } \\
\text { temperature } \\
\text { of SiNCs } \\
\left({ }^{\circ} \mathrm{C}\right)\end{array}$} & \multicolumn{2}{|c|}{$\mathrm{P}^{+} \operatorname{dose}\left(1 / \mathrm{cm}^{2}\right)$} & \multirow{2}{*}{$\begin{array}{l}\text { Annealing } \\
\text { temperature afte } \\
\text { ion implantation }\end{array}$} \\
\hline & & $100 \mathrm{keV}$ & $200 \mathrm{keV}$ & \\
\hline 1 & 1000 & & & \\
\hline 2 & 1200 & 0 & 0 & No anneal \\
\hline 3 & 1000 & & & \\
\hline 4 & 1200 & $4.0 \times 10^{13}$ & $1.0 \times 10^{14}$ & \\
\hline 5 & 1000 & & & \\
\hline 6 & 1200 & $2.0 \times 10^{14}$ & $5.0 \times 10^{14}$ & \\
\hline 7 & 1000 & & & \\
\hline 8 & 1200 & $4.0 \times 10^{14}$ & $1.0 \times 10^{15}$ & \\
\hline 9 & 1000 & & & \\
\hline 10 & 1200 & $4.0 \times 10^{15}$ & $1.0 \times 10^{16}$ & \\
\hline 11 & 1000 & & & \\
\hline 12 & 1200 & $4.0 \times 10^{16}$ & $1.0 \times 10^{17}$ & $1000^{\circ} \mathrm{C}$ \\
\hline
\end{tabular}

\section{RESULTS AND DISCUSSION}

The samples including SiNCs doped with peak P concentrations from $5 \times 10^{18}$ to $5 \times 10^{21} / \mathrm{cm}^{3}$ were systematically measured by PL at RT and ESR at $4.2 \mathrm{~K}$. Figure 2 shows (a) PL spectra for $\mathrm{SiNCs} / \mathrm{SiO}_{2}$ system with a $\mathrm{P}$ concentration of $5 \times 10^{20} / \mathrm{cm}^{3}$ (sample 9) and (b) ESR spectra for that with $5 \times 10^{18} \mathrm{P} / \mathrm{cm}^{3}$ (sample 3). As an example, sample 3 was formed by (1) $1000{ }^{\circ} \mathrm{C}$ annealing of $\mathrm{SiO}_{x}$ films, (2) double $\mathrm{P}^{+}$ion implantation at an energy of $100 \mathrm{keV}$ with a dose of $4.0 \times 10^{13} \mathrm{P}^{+} / \mathrm{cm}^{2}$ and at $200 \mathrm{kev}$ with 1.0 $\times 10^{14} / \mathrm{cm}^{2}$, (3) second annealing at $1000{ }^{\circ} \mathrm{C}$, and (4) HAT at $500{ }^{\circ} \mathrm{C}$. It can be seen in Fig. 2 that HAT enhances PL intensity by approximately ten times and decreases drastically ESR intensity of defects $\left(\mathrm{P}_{b}\right.$ centers) at the interface between $\mathrm{Si}$ core and $\mathrm{SiO}_{2}$. These results indicate that the carrier compensation by the interface defects can be almost completely removed by hydrogen passivation at $500{ }^{\circ} \mathrm{C}$. However, for these samples we were possible to detect no ESR signals of conduction electrons originated from P donors electrically activated in SiNCs because of very low spin numbers. For the samples used in our previous ESR work (Ref. 3), the volumes $\left(\sim 35 \mu \mathrm{m} \times 30 \mathrm{~cm}^{2}\right)$ of the $\mathrm{SiO}_{2}$ films with SiNCs were approximately $10^{4}$ times larger than those $\left(\sim 0.15 \mu \mathrm{m} \times 0.5 \mathrm{~cm}^{2}\right)$ of the present samples.

Even for unimplanted samples, PL intensity increased by

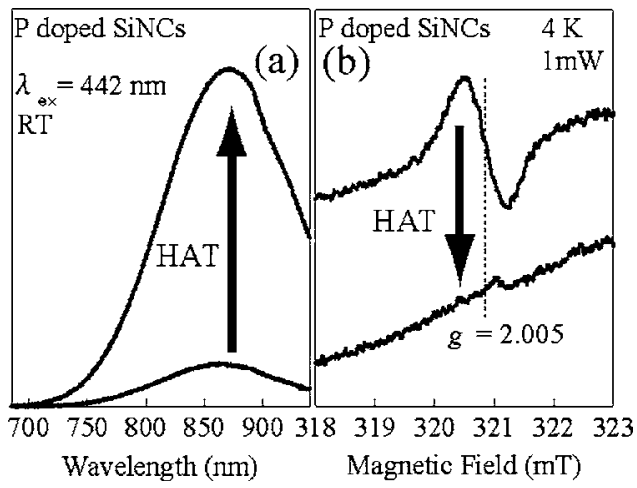

FIG. 2. HAT effects of (a) PL spectra observed at RT for sample 9 and (b) ESR spectra taken at $4.2 \mathrm{~K}$ for sample 3 . 


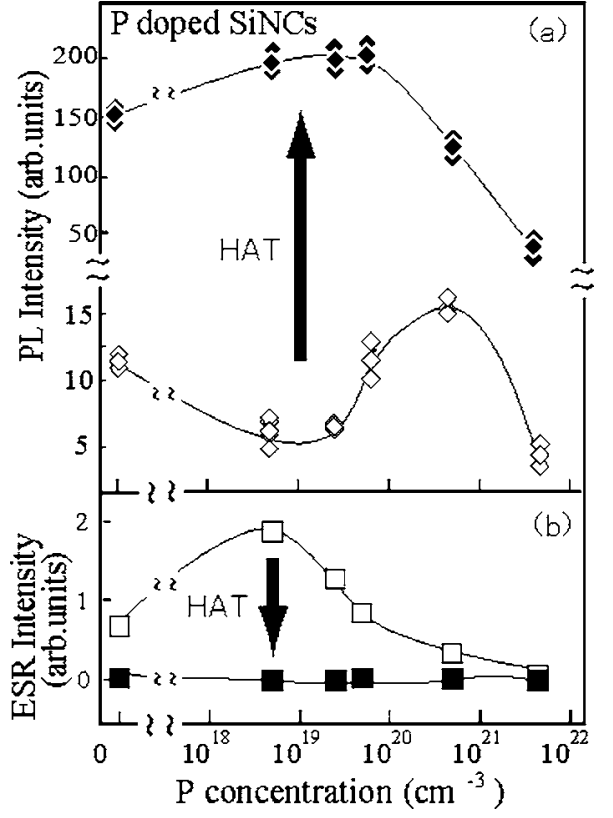

FIG. 3. Phosphorus concentration dependence of (a) the intensity of PL originated from SiNCs and (b) ESR intensity of interface defects before and after HAT.

12 times and ESR intensity decreased significantly by HAT. Similar effects were also observed for the other samples with other $\mathrm{P}$ concentrations. This strongly suggests that $\mathrm{P}$ donor concentration dependence should be investigated using $\mathrm{SiNCs} / \mathrm{SiO}_{2}$ systems in which interface defects are enough annihilated by a passivation technique such as HAT. In the previous studies (for example, Refs. 3 and 7), no attention has been paid to about nearly perfect passivation of interface defects.

Figure 3 shows the $\mathrm{P}$ concentration (or $\mathrm{P}$ dose) dependence of PL intensity for SiNCs samples (samples 1, 3, 5, 7, 9, and 11) with and without HAT, respectively. Here we assume the samples implanted with $\mathrm{P}$ ions have a homogeneous distribution of $\mathrm{P}$ between the $\mathrm{Si}$ cores and the $\mathrm{SiO}_{2}$ phase even after annealing, to describe averaged $\mathrm{P}$ concentrations instead of implanted $\mathrm{P}$ doses. These values are rough estimation because there will be competing processes, i.e., (1) the segregation coefficient $\mathrm{N}_{\mathrm{Si}} / \mathrm{N}_{\mathrm{SiO} 2}$ of $\mathrm{P}$ impurity between $\mathrm{Si}$ and $\mathrm{SiO}_{2}$ is higher than 1.0 [1.1-1.5 for thermal oxidation of bulk Si at $1000{ }^{\circ} \mathrm{C}$ (Ref. 13)] and (2) there should be $\mathrm{P}$ segregation just at the $\mathrm{Si} / \mathrm{SiO}_{2}$ interfaces for SiNCs embedded in $\mathrm{SiO}_{2} \cdot{ }^{14,15}$ For the samples without HAT, we can divide the $\mathrm{P}$ concentration into three ranges. That is, (1) the PL intensity decreases compared to that of the unimplanted sample in the lower concentration range (samples 3 and 5), (2) it increases with increasing the concentration in the intermediate range (samples 7 and 9), and (3) it decreases again with increasing the $\mathrm{P}$ concentration in the higher range (sample 11). It is noted that large enhancement of PL can be seen after HAT in the three ranges.

An ion with a high energy of 100 or $200 \mathrm{keV}$ penetrates into a $\mathrm{SiO}_{2}$ matrix including $\mathrm{SiNCs}$, and then collides with atoms to be displaced by knock-on, resulting in high density of defects of $\mathrm{SiNCs}$ and $\mathrm{SiO}_{2}$. As the ion dose increases, the SiNCs become amorphous ${ }^{6}$ and then vague via mixing in-

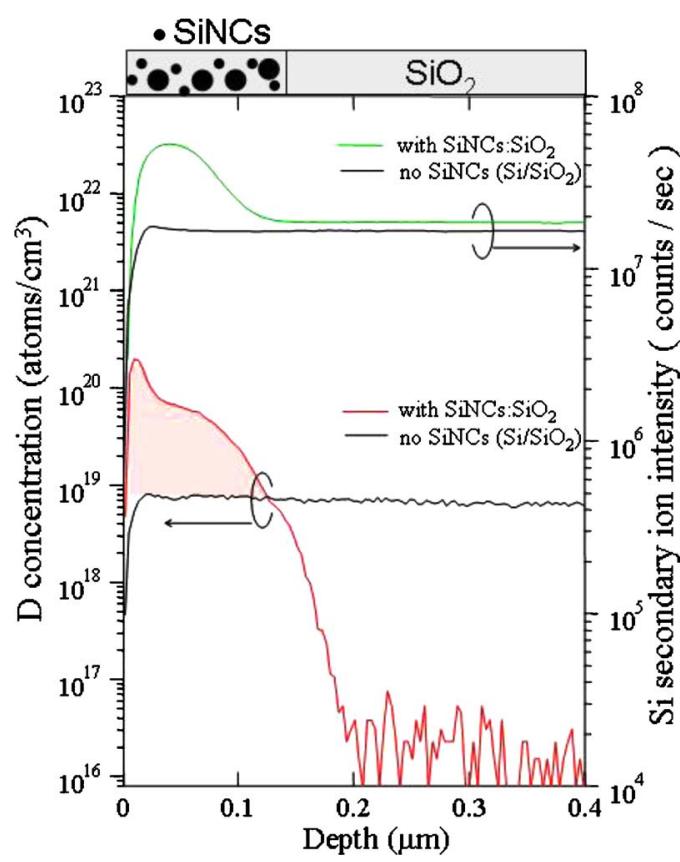

FIG. 4. (Color online) Deuterium atom profile after DAT of $\mathrm{SiO}_{2}$ layer including SiNCs obtained by SIMS measurement.

duced by the multiple knock-on phenomena. Above a critical dose (approximately $100 \mathrm{keV} \mathrm{P} \mathrm{P}^{+} \quad 4.0 \times 10^{15} / \mathrm{cm}^{2}$ $+200 \mathrm{keV} \mathrm{P} \mathrm{P}^{+} 1.0 \times 10^{16} / \mathrm{cm}^{2}$ ), complete mixing among SiNCs and $\mathrm{SiO}_{2}$ matrix will take place, leading again to a state of $\mathrm{SiO}_{x}$.

It is found from these results that some types of defects that act as nonradiative recombination centers remain even after high-temperature $\left(1000{ }^{\circ} \mathrm{C}\right)$ annealing of implanted $\mathrm{SiNCs} / \mathrm{SiO}_{2}$ system. Therefore, the defects should be passivated by an effective technique such as hydrogen passivation to obtain defect-free $\mathrm{SiNCs}$ in $\mathrm{SiO}_{2}$ for the study of ideal physical properties of SiNCs.

HAT is found to induce drastic changes in the intensities of PL and ESR spectra, as seen in Figs. 2 and 3. Similar results have been reported for hydrogen gas annealing, ${ }^{12,16}$ although the passivation effect of HAT is stronger than that of $\mathrm{H}_{2}$ gas annealing. ${ }^{12}$ Through hydrogen passivation of defects by HAT, $\mathrm{P}_{b}$ centers can be nearly eliminated, as can be seen in Fig. 3(b). We measured furthermore deuterium (D) concentration depth profile instead of hydrogen by secondary ion mass spectrometry (SIMS) for a sample after deuterium atom treatment (DAT) of a thermally oxidized $\mathrm{SiO}_{2}$ layer. The $\mathrm{SiO}_{2}$ layer with $2.0 \mu \mathrm{m}$ thickness includes high density of SiNCs formed by high dose $\left(1 \times 10^{17} / \mathrm{cm}^{2}\right) \mathrm{Si}^{+}$ion implantation followed by high-temperature annealing. Figure 4 shows SIMS profile of D as a function of depth from the surface of $\mathrm{SiO}_{2}$ layer. $\mathrm{D}$ atoms are found to be trapped by the interfaces and $\mathrm{SiO}_{2}$ layer within the $\mathrm{Si}$ ion-implanted range $(\sim 150 \mathrm{~nm})$, while $\mathrm{D}$ atoms penetrate easily into $\mathrm{SiO}_{2}$ without high density of SiNCs. If all the implanted $\mathrm{Si}$ atoms are assumed to form SiNCs with an averaged size of $5 \mathrm{~nm}$, each SiNC can be estimated to get approximately $30 \mathrm{D}$ atoms. This number suggests HAT and DAT passivate interface defects or annihilate defect levels in the band gap of SiNCs very efficiently. 


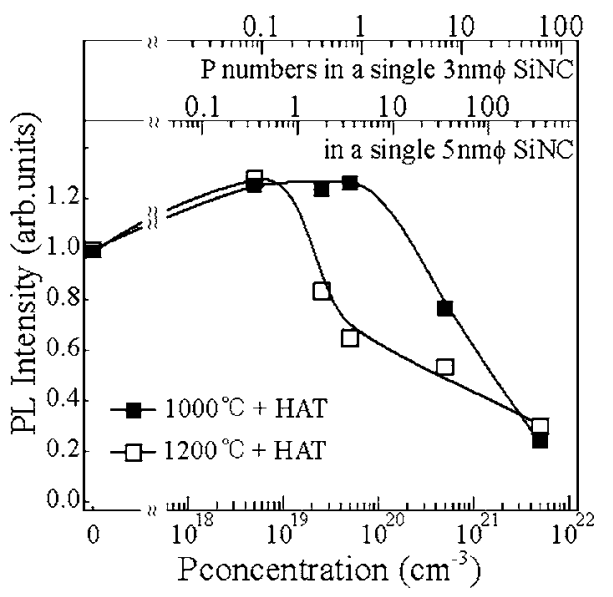

FIG. 5. PL intensity as a function of $\mathrm{P}$ peak concentration for $1000{ }^{\circ} \mathrm{C}$-annealed and $1200{ }^{\circ} \mathrm{C}$-annealed samples, followed by HAT at $500{ }^{\circ} \mathrm{C}$. The calculated, average number of $\mathrm{P}$ donors included in each SiNC with averaged diameters of $3 \mathrm{~nm}$ and $5 \mathrm{~nm}$ are shown in horizontal axes at the top, respectively.

Moreover, the PL intensity increases initially and then begins to decrease at a critical concentration as the $\mathrm{P}$ concentration increases, as can be seen in Fig. 3(a). This indicates that $\mathrm{P}$ doping in SiNCs induces small increase in PL intensity at the initial stage of lower $\mathrm{P}$ concentrations. Furthermore, it is clear that the PL intensity decreases monotonically with $\mathrm{P}$ doses higher than a critical one. This decrease is due to the Auger recombination process among excited electrons-holes, and the conduction electrons originated from donors doped in each SiNC, inducing nonradiative recombination. ${ }^{7}$

We have investigated the similar $\mathrm{P}$ concentration dependence and hydrogen passivation effects for $\mathrm{SiNCs} / \mathrm{SiO}_{2}$ systems (samples 2, 4, 6, 8, 10, and 12) obtained by $1200{ }^{\circ} \mathrm{C}$ annealing as well. This higher annealing temperature produces SiNCs with larger averaged diameters (5-6 nm) in $\mathrm{SiO}_{2}$ (see Fig. 1). In Fig. 5 the $\mathrm{P}$ concentration dependence of $\mathrm{PL}$ intensity is shown for both the $1000{ }^{\circ} \mathrm{C}$ - and $1200{ }^{\circ} \mathrm{C}$-annealed samples followed by HAT. The PL of SiNCs can be seen to increase first with increasing $\mathrm{P}$ concentration and reaches the maximum. The Auger recombination leading to the decrease in PL is clearly observed to take place above the critical $\mathrm{P}$ concentrations. The maximum can be seen at approximately $5 \times 10^{19} \mathrm{P} / \mathrm{cm}^{3}$ for $1000{ }^{\circ} \mathrm{C}$-annealed sample and $5 \times 10^{18} \mathrm{P} / \mathrm{cm}^{3}$ for $1200{ }^{\circ} \mathrm{C}$-annealed one. Thus the $\mathrm{P}$ concentrations for PL maximum depend on the averaged size of SiNCs.

These hydrogen passivation effects and $\mathrm{P}$ concentration dependence for the PL intensity of SiNCs suggest that the small increase in the PL intensity seen at relatively low $\mathrm{P}$ concentrations less than $1 \times 10^{19} \mathrm{P} / \mathrm{cm}^{3}$ is not caused by $\mathrm{P}$-atom effects ${ }^{7,15}$ of decreasing nonradiative interface defects and also of changing significantly interface structures of $\mathrm{SiNCs} / \mathrm{SiO}_{2}$. If we assume for simplicity that $\mathrm{P}$ impurities are distributed uniformly in the $\mathrm{SiNCs} / \mathrm{SiO}_{2}$ films, as described already, almost all SiNCs contain one P donor at the critical concentrations of about $5 \times 10^{19}$ and 1 $\times 10^{19} \mathrm{P}^{+} / \mathrm{cm}^{3}$ for the SiNCs formed by 1000 and $1200{ }^{\circ} \mathrm{C}$ annealing, respectively. Their averaged diameters are here estimated to be approximately 3.0 and $5.0 \mathrm{~nm}$ for each
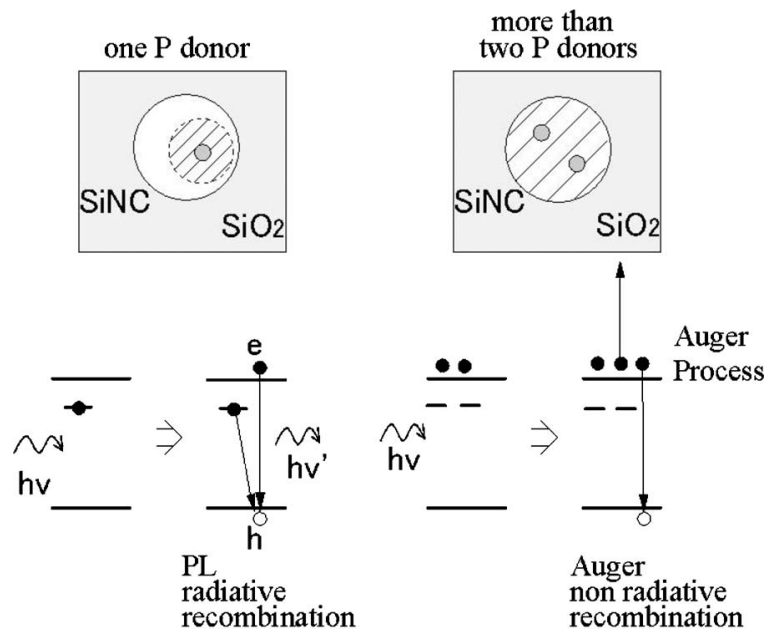

FIG. 6. A possible idea of the recombination of excited electron and hole in each SiNC including a single $\mathrm{P}$ donor and $\mathrm{P}$ donors more than two, corresponding to enhancement and quenching of PL.

sample. Below these critical concentrations, $\mathrm{P}$ concentration is not so high (in $10^{18}-10^{19} \mathrm{P} / \mathrm{cm}^{3}$ range) for changing the interface structures.

This suggests a possibility that a single $\mathrm{P}$ donor doped in each $\mathrm{SiNC}$ enhances radiative recombination process via the donor level, relatively deeper than that in bulk $\mathrm{Si}$, although this is in contrast to the conventional idea that the presence of one active donor doped in a SiNC gives Auger nonradiative recombination, leading to quenching of PL. The donor level in SiNCs has been known to be a little bit deeper $(\sim 60 \mathrm{meV})$ than that $(\sim 40 \mathrm{meV})$ of bulk crystal $\mathrm{Si}$ from larger ESR splitting of the hyperfine structure of P donors in SiNCs. ${ }^{3,4}$ Since the numbers of $\mathrm{Si}$ atoms contained in each SiNC with diameters of 3 and $5 \mathrm{~nm}$ are estimated to be approximately 680 and 3100, the number of allowed levels near the bottom of the discrete conduction band of each $\mathrm{SiNC}$ is much smaller compared to the states near the bottom of the continuum conduction band of bulk Si. Consequently a fraction of donor electrons is still bound by donor levels in SiNCs even at RT, which is entirely different from donors in bulk $\mathrm{Si}$. Here we present one of possible ideas, as shown in Fig. 6, which indicates the radiative recombination process via the donor level after electron-hole excitation when only one $\mathrm{P}$ donor is doped in each SiNC. On the other hand, more than two donor electrons tend to be far each other due to the Coulomb repulsion and so extend in $\mathrm{D}^{-}$level or the conduction band of a SiNC if two or more active $\mathrm{P}$ donors are doped in it without any compensating defects ${ }^{3}$ (see Fig. 6.) In this case, the Auger recombination process takes place more efficiently after the excitation, resulting in nonradiative recombination of excited electron-hole pair. ${ }^{7}$ It is noted that this possibility was first considered from the experimental results for the wide-range P-doped $\mathrm{SiNCs} / \mathrm{SiO}_{2}$ systems obtained by optimum hydrogen passivation of the defects at the interfaces between $\mathrm{SiNCs}$ and the surrounding $\mathrm{SiO}_{2}$ layer.

\section{CONCLUSIONS}

We have investigated (1) $\mathrm{P}^{+}$implantation effects in the two types of SiNCs (with averaged diameters of approxi- 
mately 3-4 and 5-6 nm) embedded in $\mathrm{SiO}_{2}$, followed by annealing at $1000{ }^{\circ} \mathrm{C}$, and (2) hydrogen passivation effects of the samples. Some types of defects such as $\mathrm{P}_{b}$ centers remain at the interfaces between $\mathrm{SiNCs}$ and the surrounding $\mathrm{SiO}_{2}$ even after high-temperature annealing of defects produced by ion implantation. It was clarified that hydrogen passivation using HAT induces significant changes in observed PL and ESR spectra since the interface defects are passivated with hydrogen. In wide-range $\mathrm{P}$ donor concentration dependence of PL for hydrogen passivated samples, PL intensity increases initially as the $\mathrm{P}$ concentration increases, and then begins to decrease at a critical concentration due to Auger nonradiative recombination. This suggests a possibility that a single $\mathrm{P}$ donor doped in each SiNC enhances radiative recombination process via a donor level.

\section{ACKNOWLEDGMENTS}

We would like to thank Professor N. Kishimoto for preparation of the $\mathrm{Si}^{+}$ion-implanted $\mathrm{SiO}_{2}$ samples for comparison. This work was supported in part by the "Nanoscience Research Project" of University of Tsukuba in 20022006, by METI's Innovation Research Project on Nanoelectronics Materials and Structures (2007-2010), and by MST (Foundation for Promotion of Material Science and Technology of Japan) Collaboration-Research Fund (20072008).
${ }^{1}$ P. G. Kik and A. Polman, Appl. Phys. Lett. 91, 534 (2002).

${ }^{2}$ M. Carrada, A. Wellner, V. Paillard, C. Bonafos, H. Coffin, and A. Claverie, Appl. Phys. Lett. 87, 251911 (2005).

${ }^{3}$ M. Fujii, A. Mimura, S. Hayashi, Y. Yamamoto, and K. Murakami, Phys. Rev. Lett. 89, 206805 (2002).

${ }^{4}$ D. V. Melnikov and J. R. Chelikowsky, Phys. Rev. Lett. 92, 046802 (2004).

${ }^{5}$ Y. Liu, T. P. Chen, L. Ding, M. Yang, J. I. Wong, C. Y. Ng, S. F. Tu, Z. X. Li, C. Yen, F. R. Zhu, M. C. Tan, and S. Fung, J. Appl. Phys. 101, 104306 (2007).

${ }^{6}$ D. Pacifici, E. C. Moreira, G. Franzo, V. Martorino, F. Priolo, and F. Iacona, Phys. Rev. B 65, 144109 (2002).

${ }^{7}$ A. Mimura, M. Fujii, S. Hayashi, D. Kovalev, and F. Koch, Phys. Rev. B 62, 12625 (2000).

${ }^{8}$ See, for example, Hydrogen in Semiconductors, edited by J. I. Pankove and N. M. Johnson, Semiconductors and Semimetals Vol. 34 (Academic, New York, 1991).

${ }^{9}$ A. Stesmans, Phys. Rev. B 61, 8393 (2000).

${ }^{10}$ N. Fukata, J. Chen, T. Sekiguchi, N. Okada, K. Murakami, T. Tsurui, and S. Ito, Appl. Phys. Lett. 89, 203109 (2006).

${ }^{11}$ N. Fukata, J. Chen, T. Sekiguchi, S. Matsushita, T. Oshima, N. Uchida, K. Murakami, T. Tsurui, and S. Ito, Appl. Phys. Lett. 90, 153117 (2007).

${ }^{12}$ N. Fukata, C. Li, H. Morihiro, K. Murakami, M. Mitome, and Y. Bando, Appl. Phys. A: Mater. Sci. Process. 84, 395 (2006); N. Fukata, S. Sasaki, S. Fujimura, H. Haneda, and K. Murakami, Jpn. J. Appl. Phys., Part 1 35, 3937 (1996).

${ }^{13}$ H. F. Wolf, Silicon Semiconductor Data (Pergamon, New York, 1969), p. 571 .

${ }^{14}$ K. Inoue, F. Yano, A. Nishida, T. Tsunomura, T. Toyama, Y. Nagai, and M. Hasegawa, Appl. Phys. Lett. 92, 103506 (2008).

${ }^{15}$ X. D. Pi, G. Gresback, R. W. Liptak, S. A. Campbell, and U. Kortshagen, Appl. Phys. Lett. 92, 123102 (2008).

${ }^{16}$ A. R. Wilkinson and R. G. Elliman, Appl. Phys. Lett. 83, 5512 (2003). 